DE9 1001717

\title{
Mesh Considerations for Finite Element \\ Structural Dynamic Applications
}

\author{
Ralph Robert Sterens
}

\section{DISCLAIMER}

This report was prepared as an account of work sponso, ed by an agency of the United States Government. Neither the United States Government nor any agency thureof, nor any of their employees, makes any warranty, express or implied, or assumes any legal liability or responsibility for the accuracy, completeness, or uscfulness of any information, apparatus, product, or process disclosed, or represents that its use would not infringe privately owned rights. Reference herein to any specific commercial product, process, or service by trade name, trademark, manufacturer, or otherwise does not necessarily constitute or imply its endorsement, recommendation, or favoring by the United States Government or any agency thereof. The views and opinions of authors expressed herein do not necessarily state or reflect those of the United States Government or any agency thereof.

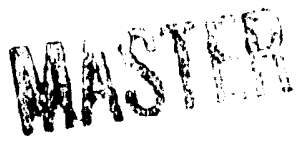




\section{CONTENTS}

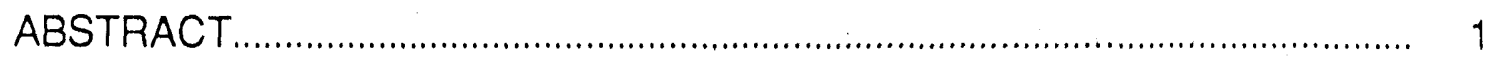

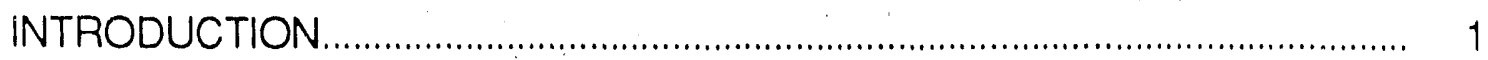

CONE FRUSTUM

SPHERE OCTANT

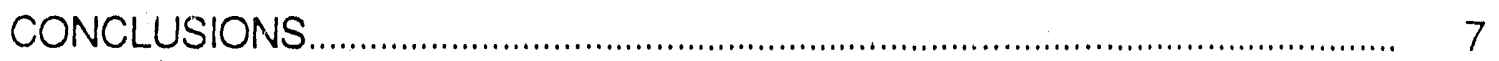

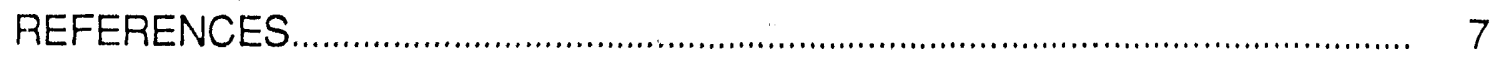

APPENDIX A. PATRAN GEOMETRIC ACCURACY ................................ 8

APPENDIX B. MODE SHAPE CORRELATION .................................. 13

APPENDIX C. SPHERE CCTANT MESH COMPARISON........................ 17

\section{FIGURES}

1. Comparison of $n=0 m=2$ mode shapes. ..................................... 3

B-1 Node-by-node mode shape comparison. ..................................... 16

C-1 Five mesh schemes for a sphere octant . ................................. 18

C-2 Two natural modes of vibration for a sphere. .......................... 21

C-3 Comparison of two mode shapes for a sphere. ......................... 24 


\title{
MESH CONSIDERATIONS FOR FINITE ELEMENT STRUCTURAL DYNAMIC APPLICATIONS
}

by

Ralph Robert Sievens

ABSTRACT

\begin{abstract}
Three factors relating to a finite element mesh and discretization scheme are discussed: the geometric accuracy of the node locations, the amount of warp of a quadrilateral shell element, and the directional biasing effect of similarly oriented triangular elements. These factors can have a significant effect on the accuracy of a finite element structural dynamic analysis. Two finite element models that illustrate these effects are described.
\end{abstract}

\section{INTRODUCTION}

Many factors affect the accuracy of finite element method (FEM) solutions. One class of factors may be called "mesh-related factors": those factors which elate to the finite element mesh and discretization scheme. For example, mesh density (number of elements in a given region) and element aspect ratio (ratio of an element's iongest length to its shortest length) are two mesh factors that can strongly affect the accuracy of the solution with shell elements. The choice of a good mesh density depends on the analyst's experience and the observed convergence of the solution with increasing density. (The convergence of several shell elements with increasing mesh densities is described by Butler and Stevens. 1)

Other mesh related factors are not as obvious as the mesh density, but miay, in some situations, lead to significant error in solution accuracy with shell eiements. Three examples are considered here: geonetric accuracy of node locations, quadrilateral element warp, and triangular element biasing. When a finite element preprocessor is used to create the FEM mesh, these factors can be overlooked. 
However, without attention to these factors, the resulting mesh may be the cause of surprisingly poor solution accuracy.

Two examples of shell element structural models that were found to be sensitive to these mesh factors will be discussed. The first model was a cone frustum. For the intended application, two planes of symmetry were used, resulting in a one-quarter model. The second model was an octant of a sphere (with symmetry and/or antisymmetry boundary conditions on the three edges). These models employed the linear shell elements TRIA2 (3-node triangular) and QUAD4 (4-node quadrilateral) in the finite element code NASTRAN. The performance of the meshes was judged by comparing vibration mode shapes and computed scattered pressures. Either a theoretical solution, an FEM solution with a much finer mesh, or a nonfinite-element refe:ence was available for all tests to judge the accuracy of the solutions.

\section{CONE FRUSTUM}

The mode shape of the $n=0, m=2$ mode (where $n$ is the circumferential mode number and $\mathrm{m}$ is the longitudinal mode number) is shown in Fig. 1a. Typical of shell structures of this type, the modal density was high: in this example, at least four $n>0$ modes existed within $1 \%$ of the frequency of the desired mode.

When the eigenvalue problem was solved with the initial mesh, no $n=0, m=2$ mode was found. The mode with a frequency closest to the frequency of the true $n=0, m=2$ mode showed a significant $n=0, m=2$ component, but it was "contaminated" with higher $n$ and $m$ modes (see Fig. 1b). The convergence of the eigensolution was not the source of the poor mode shape; different eigenvalue solution methods within NASTRAN, and even a different finite element code (using the same mesh, but a different element), yielded a similar mode shape. The "true" frequency and mode shape were determined by two methods: a finite element model wiih a much finer mesh and an axisymmetric nonfinite element method. These methods showed excellent agreement with each other, both in frequency and in mode shapes.

The cause of this poor accuracy was traced to small errors in the locations of the nodes. A finite element preprocessor (PATRAN2) was used to create the mesh; the PATRAN options that were used 


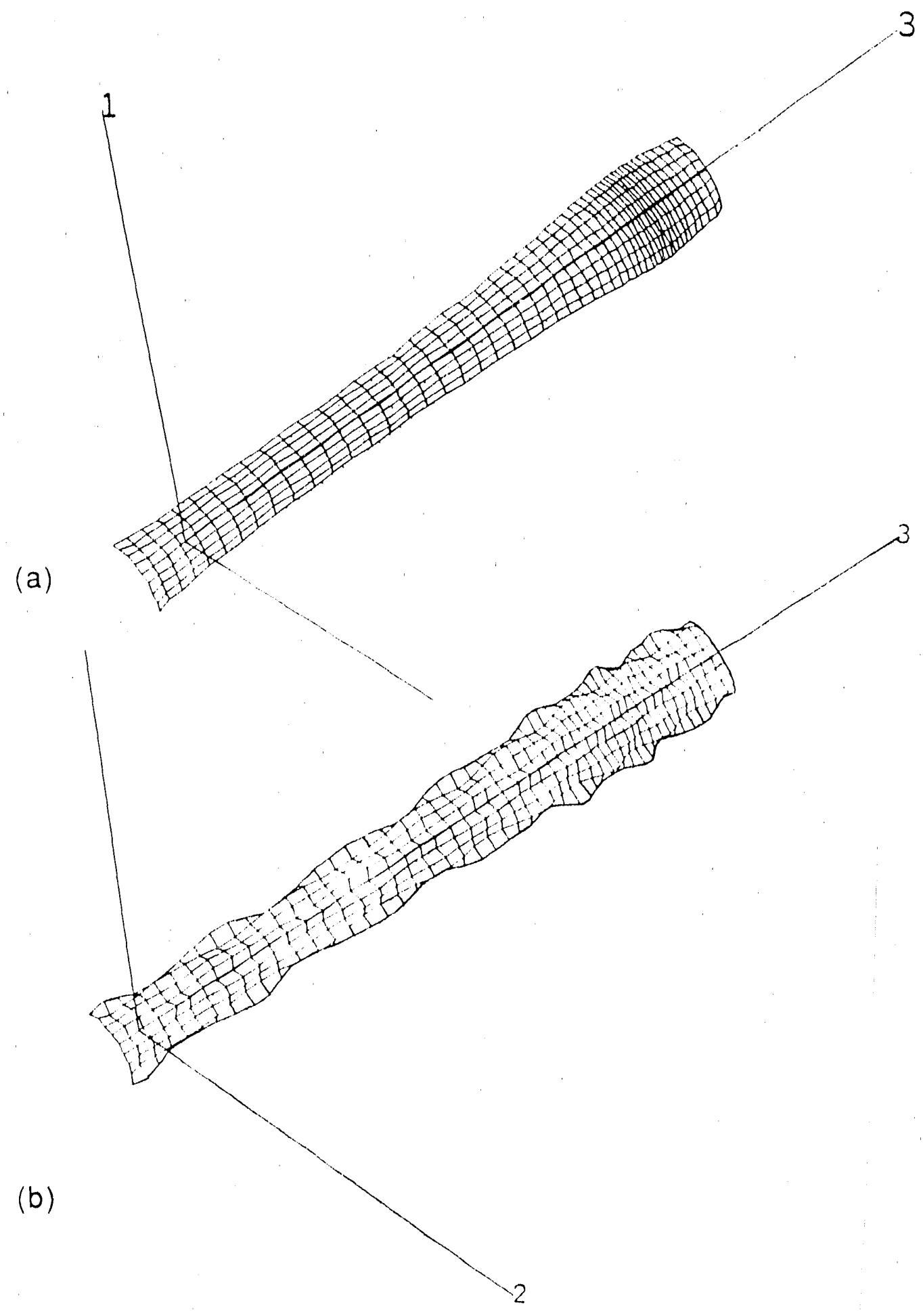

Fig. 1. Comparison of $n=0 m=2$ mode shapes:

(a) $n=0 \quad m=2$ mode, using accurately defined mesh;

(b) $n=0 \quad m=2$ mode, using imperfect mesh. 
gave errors in node locations on the order of $0.5 \%$ in the radial direction. This small deviation caused "dimples" in the otherwise perfectly conical surface, and these dimples caused the "contamination" of the calculated mode shape.

Details of the methods used to create the mesh with the preprocessor and how to avoid this type of problem are discussed in Appendix A. The important point is this: small deviations of the model's node locations from the true surface resulted in significant error in the calculated mode shape. Three other points should be noted:

- In a less symmetric structure, the small nodal inaccuracies may have been far less significant.

- The radial deviations of the nodes from the actual surface was not random: systematic trends were observed.

- Visually, the model appeared correct. Frequently, no further checks beyond a visual check are performed when using a graphic-oriented finite element preprocessor.

When the model was remeshed with no node position error (to 6 decimal places), the mode shape appeared nearly indistinguishable from the reference mode shape.

A method of quantitatively measuring the correlation between the mode shape under question and a reference mode shape was used for comparing these mode shapes. Two numbers, the modal scale factor and the mode shape correlation coefficient, were computed for the mode shape being evaluated. The mode shape correlation coefficients computed for each mode shape in this cone frustum example are shown in Table I.

Table 1

Comparison of Mode Shapes

Mesh

Mode Shape Correlation Coefficient

Fine mesh (reference)

Initial coarse mesh ("flawed")

Corrected coarse mesh ("perfect")

0.59

0.91 
The remaining mode-shape imperfection was attributed to the coarseness of the mesh. The mode-shape correlation coefficient and its statistical properties are discussed in Appendix B.

\section{SPHERE OCTANT}

An octant of a sphere is a difficult shape to mesh for several reasons. Element warp, poor element aspect ratio, nonuniform meshing density, or triangular element directional biasing can be present in many meshing schemes. To determine the "best" method of meshing a sphere octant, we considered the five meshing schemes shown in Fig. $\mathrm{C}-1$ in Appendix C. Four tests were used to compare the meshes: the far-field scattered prossure was calculated in an acoustic analysis, two axisymmetric natural modes were extracted, and a static pressure load was applied to isolate the effect of stiffness-related effects from mass-related effects.

By definition, a sphere possesses spherical symmetry. A sphere octant can be used to model a spherical shell in a linear finite element analysis by properly combining symmetry and antisymmetry boundary conditions on the octant's three edges. No finite element mesh can possess spherical symmetry because of the discrete element representation of the numerical method. However, all of the meshes considered here were axisymmetric about the $x$-axis, and all of the meshes except Meshes 1 and 4 were also axisymmetric about the $y$-and $z$-axes. All of the tests used to compare these meshes involved axisymmetric deformation about the $x$-axis. The performance of these meshes for deformation not axisymmetric with respect to the $x$-axis was not considered in this study.

The five meshes considered for the sphere octant model did not have the same number of nodes; for this reason, the comparison was not rigorous. However, the meshing schemes could not be altered to have equal numbers of nodes: e.g.. the scheme of Mesh 2 could have 61 nodes ( 8 elements per edge) or 91 nodes (10 elements per edge), but it could not have 73 nodes (to match Mesh 1). Despite this inequity, some observations can be made about the mesh schemes themselves:

- All meshes had 8 elements ( 9 nodes) along each edge. 
- Mesh 5 was constructed by simply dividing each quadrilateral of Mesh 2 into two triangles. This doubled the number of elements, but left the number and location of nodes ihe same.

- The quadrilateral elements of Meshes $i$ and 4 were flat (zero warp); those of Mesh 2 were not flat. This warpage is an unavoidable consequence of the meshing scheme of this mesh.

- Triangular elements are always flat.

The results of the comparison are summarized in Appendix C. One noteworthy observation is this: although none of the meshes gave a "perfect" mode shape, the mode shapes of Meshes 1 and 4 were axisymmetric with respect to the x-axis. Meshes 2, 3, and 5 gave mode shapes that were not axisymmetric; an example of such a mode shape is shown in Fig. C-2a in Appendix C for Mesh 2. In Meshes 3 and 5 , the mode shapes were also not axisymmetric, but they were symmetric about the $x z^{\prime}$-plane (where $z^{\prime}$ is an axis at a 45-deg angle to the $y$-and z-axes in the $y z$-plane) as shown in Fig. C-2b in Appendix $C$. The cause of this lack of axisymmetry is attributed to element warp in the case of Mesh 2; in the case of Meshes 3 and 5, it is attributed to the systematic pattern of the triangular elements (triangle directional biasing). The lack of axisymmetry in the mode shapes was reflected in the lower correlation coefficients for these modes, as shown in Table 11.

Table $\|$

Mode Shape Correlation Coefficients

\begin{tabular}{ccc} 
Mesh & Mode 1 & Mode 2 \\
\hline 1 & 0.999 & 0.977 \\
2 & 0.997 & 0.825 \\
3 & 0.995 & 0.770 \\
4 & 0.999 & 0.985 \\
5 & 0.879 & 0.406
\end{tabular}




\section{CONCLUSIONS}

Geometric accuracy of node locations, quadrilateral element warp, and triangular element biasing are three mesh-related problems that can cause significant errors in some FEM shell dynamic analyses. The cone frustum and sphere octant models illustrated these effects. These meshing problems can be inadvertently caused by a finite element preprocessor or can be an unavoidable consequence for some mesh scheme/structure surface combinations (e.g., sphere octant Mesh 2). Triangular element directional biasing may be difficult to avoid in many mesh schemes.

The significance of these effects may be greater for some structures (e.g., symmetric structures) than for others, but the fact that they can lead to substantial errors makes them worth attention.

A statistical method for quantitatively comparing two mode shapes was employed in the analysis of the mode shapes of the example structures. This method gives a measure of the correlation between a reference mode shape (taken to be the "true" value) and a mode shape under test; it is a convenient way to judge the accuracy of a mode when a reference is available.

\section{REFERENCES}

1. T. A. Butler and R. R. Stevens, "Shell Element Evaluation," Los Alamos National Laboratory document, May-September, 1989.

2. PATRAN Plus User's Manual, Release 2.4, PDA Engineering, PATRAN Division, Costa Mesa, California, 1989. 
APPENDIX A

PATRAN GEOMETRIC ACCURACY 


\section{PATRAN GEOMETRIC ACCURACY}

It has been observed that finite element meshes made with PATRAN may contain some errors in the location of nodes, i.e., the nodal coordinates that PATRAN computes are not exactly where the user intended them to be. Such errors have been observed in arcs (lines) and surfaces.

In some cases, the errors arise because PATRAN uses third-order (cubic) parametric equations to approximate all shapes. This approximation will always give errors for shapes that cannot be exactly described by a cubic equation (e.g., circular arcs). In other cases, the sources of PATRAN's errors are not known.

Two cases of typical mesh-generating methods that can create small errors in nodal locations are described below. These cares are not exhaustive: other methods may also create nodal location errors.

\section{A. Case One: Accuracy of Circular Arcs}

Four circular arcs were constructed (using the PATRAN ARC linegeneration command), with arc lengths of $90,45,22.5$, and 11.25 degrees, with a unit radius, and lying in the $x y$-plane. Nodes were then created on these lines at 5.625-degree intervals. The nodal coordinates for these four line segments are given in Table 1.

Observations:

(1). The radial distance of all nodes is very close to the intended radius.

(2). The nocles have exact coordinates at both end points and at the midpoint of the arc. The $x$ - and $y$-coordinates of the other nodes are in error: the greater the arc length, the greater the error.

(3). Although the nodes are out of proper position, they have the correct radius. This is probably due to the parametric representation of the line, with the $x$-cooldinate going from 1.0 to 0.0 , and the $y$-coordinate going from 0.0 to 1.0 . For noncircular shapes, the radial distance error would not alway's come out zero. 


\section{B. Case Two: Accuracy of Patches (Surfaces)}

Nodes on a surface are defined by the geometry of the surface's "parent" patch. There are many ways to create a patch in PATRAN, but the general approach is to create two or more lines that define the edges of the patch.

A conical surface was defined by the two-line (2L) patch option. Arcs of various lengths were used as the two curved edges of the patch. With this method, the accuracy of the nodes created on the surface of the patch is determined by the accuracy of the two arcs, and the information above (Accuracy of Circular Arcs) applies.

Observations:

(1). The axial coordinates of all nodes are essentially exact.

(2). The nodes have exact coordinates on both straight edges and on the straight midcurve line of the surface.

(3). The $x$ - and $y$-coordinates (coordinates in the plane of the cone's perpendicular cross section) of the other nodes are in error: the greater the arc length, the greater the error.

(4). The radial distance of all nodes is very close to the intended radius. (Again, this surface was created from two circular arcs. Noncircular arcs could give rise to radial direction errors.)

Note: Surfaces created by other patch options give rise to other errors. 


\section{Table 1}

\section{Nodal Coordinates for Four Line Segments}

\section{Intended coordinates:}

\begin{tabular}{llll} 
Point & $x$-Coordinate & $y$-Coordinate & $\begin{array}{l}\text { Angle } \\
\text { Ideg) }\end{array}$ \\
\cline { 2 - 3 } & & & \\
1 & 1.0 & 0.0 & 0.0 \\
2 & 0.995185 & 0.098017 & 5.625 \\
3 & 0.980785 & 0.195090 & 11.25 \\
4 & 0.956940 & 0.290285 & 16.875 \\
5 & 0.923880 & 0.382683 & 22.5 \\
6 & 0.881921 & 0.471397 & 28.125 \\
7 & 0.831470 & 0.555570 & 33.75 \\
8 & 0.773010 & 0.634393 & 39.375 \\
9 & 0.707107 & 0.707107 & 45.0 \\
points 10.17 are the mirror image of 1.9 about a 45. deg axis
\end{tabular}

(1) 90 degree arc

\begin{tabular}{|c|c|c|c|c|c|c|}
\hline Point & $x$-Coordinate & $y$-Coordinate & $\begin{array}{l}\text { Angle } \\
\text { (degl }\end{array}$ & $\begin{array}{l}\text { Radius } \\
\% \text { error })\end{array}$ & $\begin{array}{c}\text { x-Coordiriate } \\
\% \text { error) }\end{array}$ & $\begin{array}{c}y \text {-Coordinate } \\
\% \text { \%error) }\end{array}$ \\
\hline 1 & 1.0 & 0.0 & 0.0 & 0 & 0 & 0 \\
\hline 2 & 0.994837 & 0.102244 & 50.8679 & 0.0077 & .0 .0350 & 4.3125 \\
\hline 3 & 0.979684 & 0.201535 & 110.6244 & 0 & -0.1123 & 3.3036 \\
\hline 4 & 0.955042 & 0.297369 & 170.2950 & 0 & -0.1980 & 2.4404 \\
\hline 5 & 0.921415 & 0.389245 & 220.9013 & 0 & .0 .2668 & 1.7147 \\
\hline 6 & 0.879305 & 0.476659 & 280.4615 & 0 & .0 .2966 & 1.1163 \\
\hline 7 & 0.829216 & 0.559109 & 330.9903 & 0 & -0.2711 & 0.6370 \\
\hline 8 & 0.771649 & 0.636093 & 390.4998 & 0 & -0.1761 & 0.2680 \\
\hline 9 & 0.707107 & 0.707107 & 450.0 & 0 & 0 & 0 \\
\hline
\end{tabular}

points 10.17 are the mirror image of 1.9 about a 45. deg axis 
Table $1 \cdot$ con't

\section{(2) 45 degree arc}

\begin{tabular}{|c|c|c|c|c|c|c|}
\hline Point & $x$-Coordinate & $y$-Coordinate & $\begin{array}{l}\text { Angle } \\
\text { (degl) }\end{array}$ & $\begin{array}{l}\text { Radius } \\
\% \text { error }\end{array}$ & $\begin{array}{c}x \text {-Coordinate } \\
1 \% \text { errer }\end{array}$ & $\begin{array}{c}y \text {-Coordinate } \\
\text { Lerror) }\end{array}$ \\
\hline 1 & 1.0 & 0.0 & 0.0 & 0 & 0 & 0 \\
\hline 2 & 0.995107 & 0.098837 & 5.6460 & 0 & .0 .0078 & 0.8370 \\
\hline 3 & 0.980608 & 0.196001 & 11.3032 & 0 & -0.0180 & 0.4670 \\
\hline 4 & 0.956775 & 0.290835 & 16.9079 & 0 & .0 .0172 & 0.1895 \\
\hline 5 & 0.923879 & 0.382683 & 22.5000 & 0 & 0 & 0 \\
\hline
\end{tabular}

points 6-9 are the mirror image of $1-5$ about a 22.5-deg axis

(3) 22.5 degree arc

\begin{tabular}{|c|c|c|c|c|c|c|}
\hline Point & $x \cdot$ Coordinate & $y$-Coordinato & $\begin{array}{l}\text { Angle } \\
\text { (degl }\end{array}$ & $\begin{array}{l}\text { Radius } \\
\% \text { erres }\end{array}$ & $\begin{array}{c}\text { x-Coordiriate } \\
0 \% \text { error }\end{array}$ & $\begin{array}{c}y \text {-Coordinate } \\
\text { \%error }\end{array}$ \\
\hline 1 & 1.0 & 0.0 & 0.0 & 0 & 0 & 0 \\
\hline 2 & 0.995173 & 0.098134 & 5.6317 & 0 & .0 .0012 & 0.1196 \\
\hline 3 & 0.980785 & 0.195090 & 11.2500 & 0 & 0 & 0 \\
\hline
\end{tabular}
points 4 and 5 are the mirror image of 1 and 2 about an 11.25-deg axis

(4) 11.25 degree arc

\begin{tabular}{|c|c|c|c|c|c|c|}
\hline Point & $x \cdot$ Coordinate & $y$-Coordinate & $\begin{array}{l}\text { Angle } \\
(d \in d)\end{array}$ & $\begin{array}{l}\text { Radius } \\
\% \text { errer }\end{array}$ & $\begin{array}{c}x \text {-Coordinate } \\
\text { \% errers }\end{array}$ & $\begin{array}{c}\text { y" Coordinate } \\
\% \text { \%rrer }\end{array}$ \\
\hline 1 & 1.0 & 0.0 & 0.0 & 0 & 0 & 0 \\
\hline 2 & 0.995185 & 0.098017 & 5.6250 & 0 & 0 & 0 \\
\hline 3 & 0.980785 & 0.195090 & 11.2500 & 0 & 0 & 0 \\
\hline
\end{tabular}


APPENDIX B

MODE SHAPE CORRELATION 


\section{MODE SHAPE CORRELATION}

A Fortran program has been developed to numerically compare two mode shapes. This program may be extended to handle complex quantities. It is useful for quantitatively comparing an approximate or uncertain mode shape with a reference (known) mode shape. For example, a mode from a relatively coarse finite element mesh could be compared with the corresponding mode found from a finer finite element mesh, or the mode could be compared with an experimentally determined mode.

The program essentially does three things:

(1). Based on the nodal cocrdinates (required input), the closest reference node to each test node is determined.

(2). Two statistics are computed:

(a). Mode scale factor (msf): the slope of the best (in the least squares-sense) straight line fit through the points and passing through the origin:

$$
\operatorname{msf}(x, r)=\frac{\sum_{j=1}^{n}\left(\varphi_{x}\right)_{j}\left(\varphi_{r}\right)_{j}^{*}}{\sum_{j=1}^{n}\left(\varphi_{r}\right)_{j}\left(\varphi_{r}\right)_{j}^{*}},
$$

where $j_{x}$ is the "test" mode shape, $j_{r}$ is the reference mode shape, and $n$ is the number of points in the test mode shape. This parameter gives no indication of the quality of the fit of the points to the straight line, only the slope. It is affected by the normalization of the two mode shapes.

(b). Mode shape correlation coefficient (mscc): a measure of the least-squares deviation of the points from the straight line:

$$
\operatorname{mscc}(x, r)=\frac{\left|\sum_{j=1}^{n}\left(\varphi_{x}\right)_{j}\left(\varphi_{r}\right)_{j}^{*}\right|^{2}}{\sum_{j=1}^{n}\left(\varphi_{x}\right)_{j}\left(\varphi_{x}\right)_{j}^{*} \sum_{j=1}^{n}\left(\varphi_{r}\right)_{j}\left(\varphi_{r}\right)_{j}^{*}} .
$$


Note that the mscc is a real quantity, even if the reference and/or test mode shapes are complex. The mscc does not indicate whether the deviations of the two mode shapes are due to random scatter or systematic trends.

If the test and reference mode shapes are closely correlated (i.e., both mode shapes represent the same mode shape), then the mscc will have a value close to 1.0. If the two mode shapes are also normalized in the same way, then the msf will be close to 1.0. If the mode shapes are uncorrelated, the mscc will have a value close to 0.0 .

These statistics are described in more detail in Ref. 1.

(3). The two mode shapes are plotted against each other on a node-by-node basis, that is, for each pair of corresponding nodes, the reference mode shape value is plotted against the test mode shape value. In this way, if the two mode shapes were perfectly correlated, all the points plotted would lie on a straight line. If the mode shapes were normalized in the same way, the slope of the line would be unity. An example of such a plot is shown in Fig. B-1.

\section{REFERENCES}

1. D. J. Ewins, Modal Testing: Theory and Practice (Research Studies Press LTD., Letchworth, Hertfordshire, England, 1986), p. 224. 


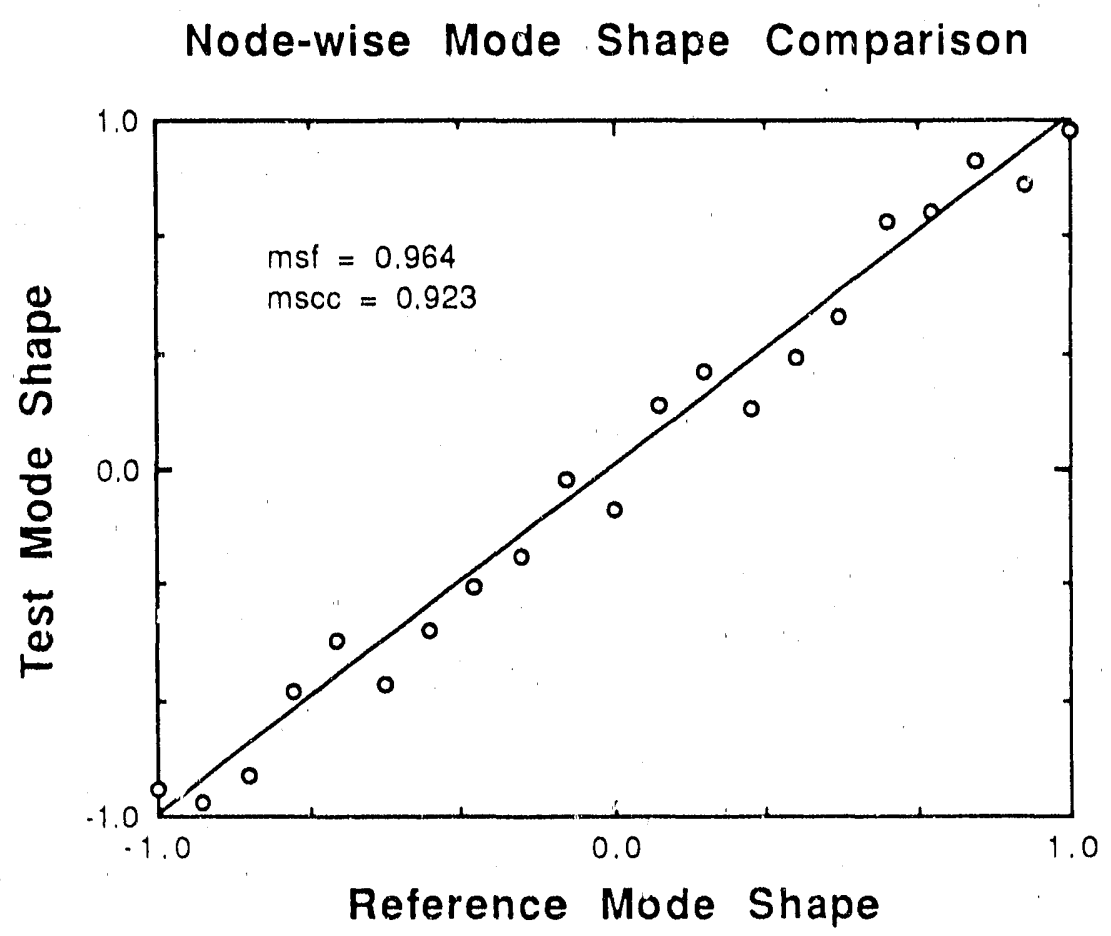

Fig. B-1. Node-by-node mode shape comparison. 


\section{APPENDIX C}

\section{SPHERE OCTANT MESH COMPARISON}




\section{SPHERE OCTANT MODELING}

The five meshes used in the sphere octant comparison are shown in

Fig. C-1. The meshing methods were compared in three ways:

(1). The scattered pressure from a plane wave (at two frequencies) was calculated and compared with an analytical solution.

(2). The frequency, generalized mass, and mode shape of two natural modes were compared with reference values.

(3). A uniform static pressure was applied, and the resulting displacement was compared with the theoretical value.

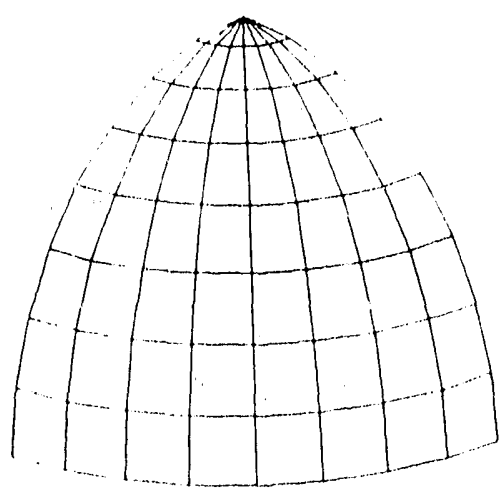

mesh 1

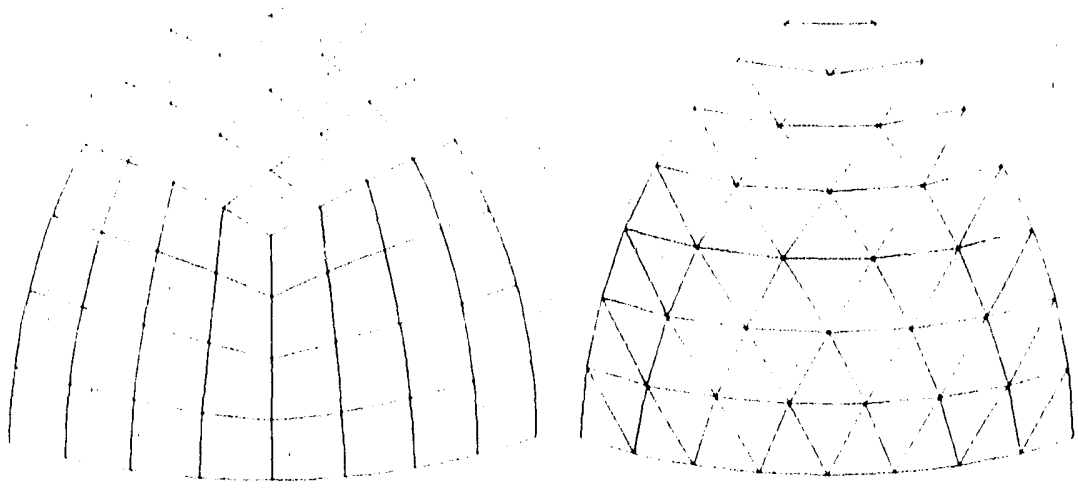

mesh 2

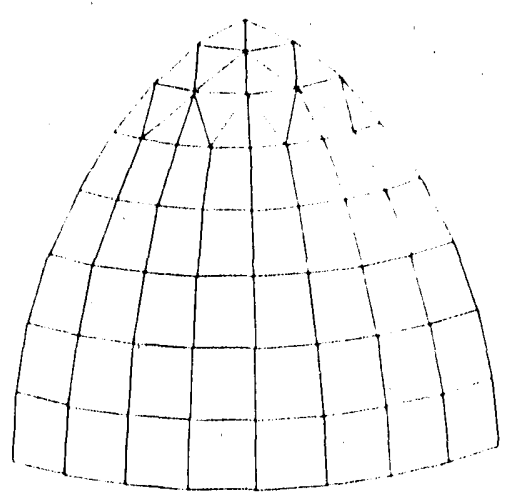

mesh 4 mesh

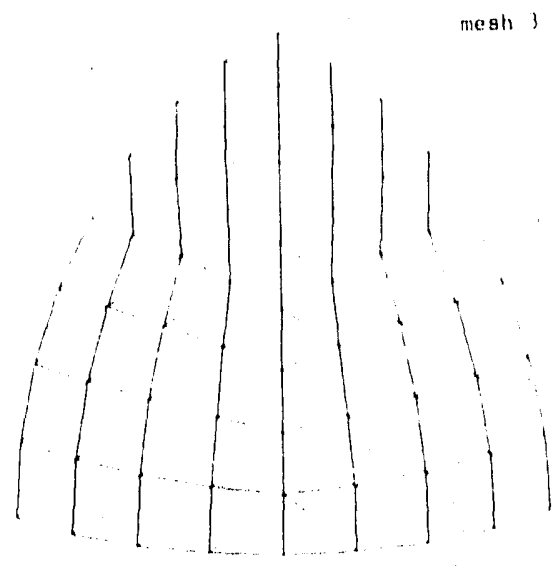

megh is

Fig. C-1. Five Mesh Schemes for a Sphere Octant. 
As shown in Table $\mathrm{C}-\mathrm{I}$, the five meshes considered all had eight elements (nine nodes) along each edge, giving varying total numbers of nodes and elements.

$\begin{array}{ccc}\text { Mesh } & \begin{array}{c}\text { Table C-1 } \\ \text { Comparison of Meshes } \\ \text { Number of } \\ \text { Nodes }\end{array} & \begin{array}{c}\text { Number of } \\ \text { Elements }\end{array} \\ \text { Mesh 1 } & 73 & 64 \\ \text { Mesh 2 } & 61 & 48 \\ \text { Mesh 3 } & 45 & 64 \\ \text { Mesh 4 } & 63 & 60 \\ \text { Mesh 5 } & 61 & 96\end{array}$

Mesh 5 was constructed by simply dividing each quadrilateral of Mesh 2 into two triangles, doubling the number of elements but keeping the number and location of nodes identical.

\section{Scattered Pressure}

The percent error (defined as FEM/analytical - 1.0) in the scattered pressure is given in Table C-II. In this table, forward refers to pressure scattered in the same direction as the incident wave, back refers to the pressure scattered backward toward the source of the wave, and side refers to the pressure scattered at a 90-deg angle to the incident wave.

$$
\begin{gathered}
\text { Table C-ll } \\
\text { Scattered Pressure Comparison }(k a=1.0) \\
\text { (Percent Error) }
\end{gathered}
$$

Eorward

Mesh 1

Mesh 2

Mesh 3

Mesh 4

Mesh 5
$-3.70$

$-0.49$

1.80

$-2.20$

$-0.39$
Back

$-2.91$

$-3.68$

$-8.17$

$-2.62$

$-3.32$
Side
0.10
$-0.54$
$-1.57$
$-0.10$
$-0.79$ 
Table C-III

Scattered Pressure Comparison $(k a=1.6)$

(Percent Error)

\begin{tabular}{rrr} 
Forward & Back & Side \\
\hline 3.14 & 3.44 & 3.42 \\
0.79 & 2.51 & 4.56 \\
-5.57 & -1.54 & 3.75 \\
214 & 3.22 & 4.46 \\
-0.73 & 1.32 & 3.82
\end{tabular}

\title{
2. Natural Mode
}

The two vibration modes that were considered were axisymmetric:

one had 4 half waves and the other 12 half waves (radial displacement) around the circumference (see Fig. C-2). The reference against which the four meshes were compared was calculated by BOSOR (a finite difference axisymmetric shell analysis program ${ }^{1}$ ). Symmetry boundary conditions were used on all cutting planes of the three-dimensional meshes to simulate the entire spherical shell. The following table summarizes the modal parameters.

\author{
Table C-IV \\ Natural Mode Comparisons
}

MODE 1

\begin{tabular}{lccc} 
Mesh & $\begin{array}{c}\text { Frequency } \\
(H z)\end{array}$ & $\begin{array}{c}\text { Generalized } \\
\text { Mass }\end{array}$ & $\begin{array}{c}\text { Mode Shape } \\
\text { Correlation Coefficient }\end{array}$ \\
\hline BOSOR & 121.63 & 12,995 & $(1.0)$ \\
Mesh 1 & 121.30 & 10,698 & 0.999 \\
Mesh 2 & 121.51 & 12,908 & 0.997 \\
Mesh 3 & 122.22 & $15,210$. & 0.995 \\
Mesh 4 & 121.35 & $11,720$. & 0.999 \\
Mesh 5 & 121.75 & $10,973$. & 0.879
\end{tabular}




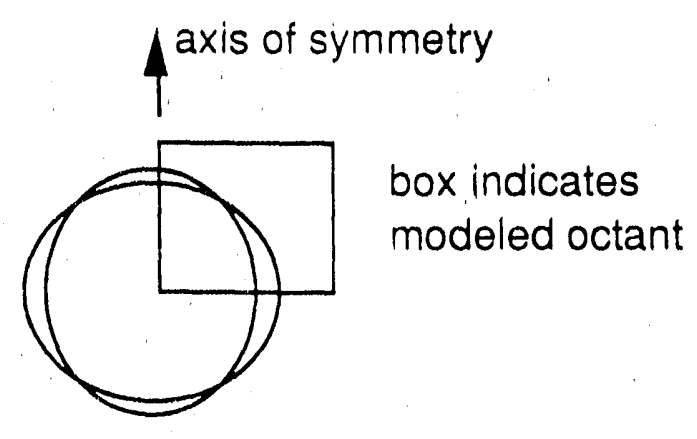

MODE 1

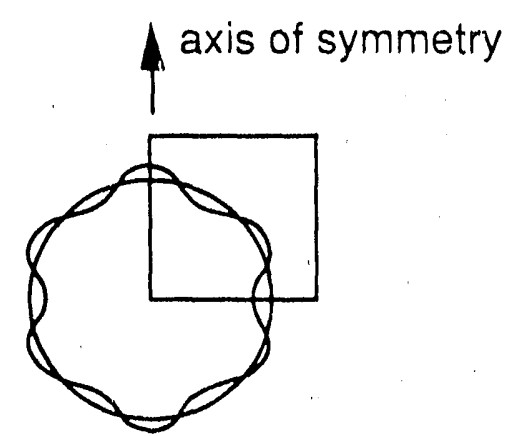

MODE 2

Fig. C-2. Two natural modes of vibration of a sphere.

\begin{tabular}{|c|c|c|c|}
\hline & NA & $\begin{array}{l}\text { Table C-V } \\
\text { L MODE COMP }\end{array}$ & RISONS \\
\hline & & MODE 2 & \\
\hline Mesh & $\begin{array}{l}\text { frequency } \\
\qquad(\mathrm{Hz})\end{array}$ & $\begin{array}{c}\text { generalized } \\
\text { Mass }\end{array}$ & $\begin{array}{l}\text { mode shape } \\
\text { Correlation Ceefficient }\end{array}$ \\
\hline BOSOR & 170.02 & 3629.88 & $(1.0)$ \\
\hline Mesh 1 & 154.34 & 3744.11 & 0.977 \\
\hline Mesh 2 & 158.53 & 4012.88 & 0.825 \\
\hline Mesh 3 & 178.50 & 8033.54 & 0.770 \\
\hline Mesh 4 & 156.79 & 3749.87 & 0.985 \\
\hline Mesh 5 & 171.56 & 4635.56 & 0.406 \\
\hline
\end{tabular}

\section{Static Pressure}

The radial displacement caused by a uniform static pressure over the surface of each mesh is given in Table C-VI. 


\section{Table C-VI \\ RADIAL DISPLACEMENT}

Mesh

$\longrightarrow$

THEORY

Mesh 1

Mesh 2

Mesh 3

Mesh 4

Mesh 5

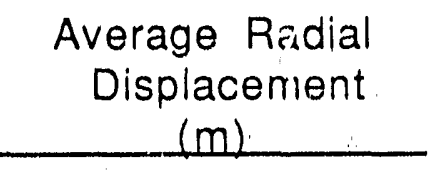

2.818 e- 10

$2.817 \quad \theta-10$

$2.794 \quad \theta-10$

$2.744 \quad \theta-10$

$2.798 \quad e-10$

$2.809 \quad 3-10$
Standard Deviation of

Radial Displacement

(m)

$(0.0)$

1.410 e- 11

$0.783 \mathrm{e}-11$

$2.502 \quad e-11$

1.006 e-11

3.375 e-11

\section{Observations}

The scattered pressure results show no clear superiority of one meshing method over another, with the exception of Mesh 3, which gave relatively poor backscattering results at $k a=1.0$. It is noteworthy that this mesh had the fewest nodes.

The data for natural frequency and natural mode shapes show an interesting result. Theoretically, the mode shapes should all be axisymmetric (i.e., all displacements of a given polar angle should be equal). Mesh 1 and Mesh 4 gave axisymmetric mode shapes, but Meshes 2, 3, and 5 gave mode shapes that were not axisymmetric. An example of an axisymmetric mode shape is shown in Fig. C-3a for Mesh 2. In Meshes 3 and 5, the mode shapes were not axisymmetric, but they were symmetric about the plane with a 45-deg angle to the $x z$-plane and containing the $x$-axis (see Fig. C-3b). This lack of axisymmetry in the mode shapes was reflected in the lower correlation coefficient values for these modes. This lack of axisymmetry occurs for both modes considered, though it is most pronounced for the higher mode.

The frequency prediction for the higher frequency mode for all meshes except Mesh 5 showed a 5 to $9 \%$ error (Mesh 5 gave only a 1 $\%$ error). However, for the lower frequency mode, which had a simpler mode shape, all meshes gave a frequency prediction within $0.5 \%$ of the reference. The higher frequency mode was considered to be a severe test for these relatively coarse meshes. 
The average radial displacements for the uniform static pressure load are ail within $3 \%$ of the theoretical value. The mesh with the least average radial deviation is Mesh 2, while Mesin 5 had the greatest deviation.

\section{REFERENCES}

1. D. Bushnell, "BOSOR 4: Program for Stress, Buckling, and Vibration of Complex Shells of Revolution," in Structural Mechanics Software Series - Vol. 1, N. Perrone and W. Pilkey, Eds. (University Press of Virginia, Charlottesville, Virginia, 1976). 


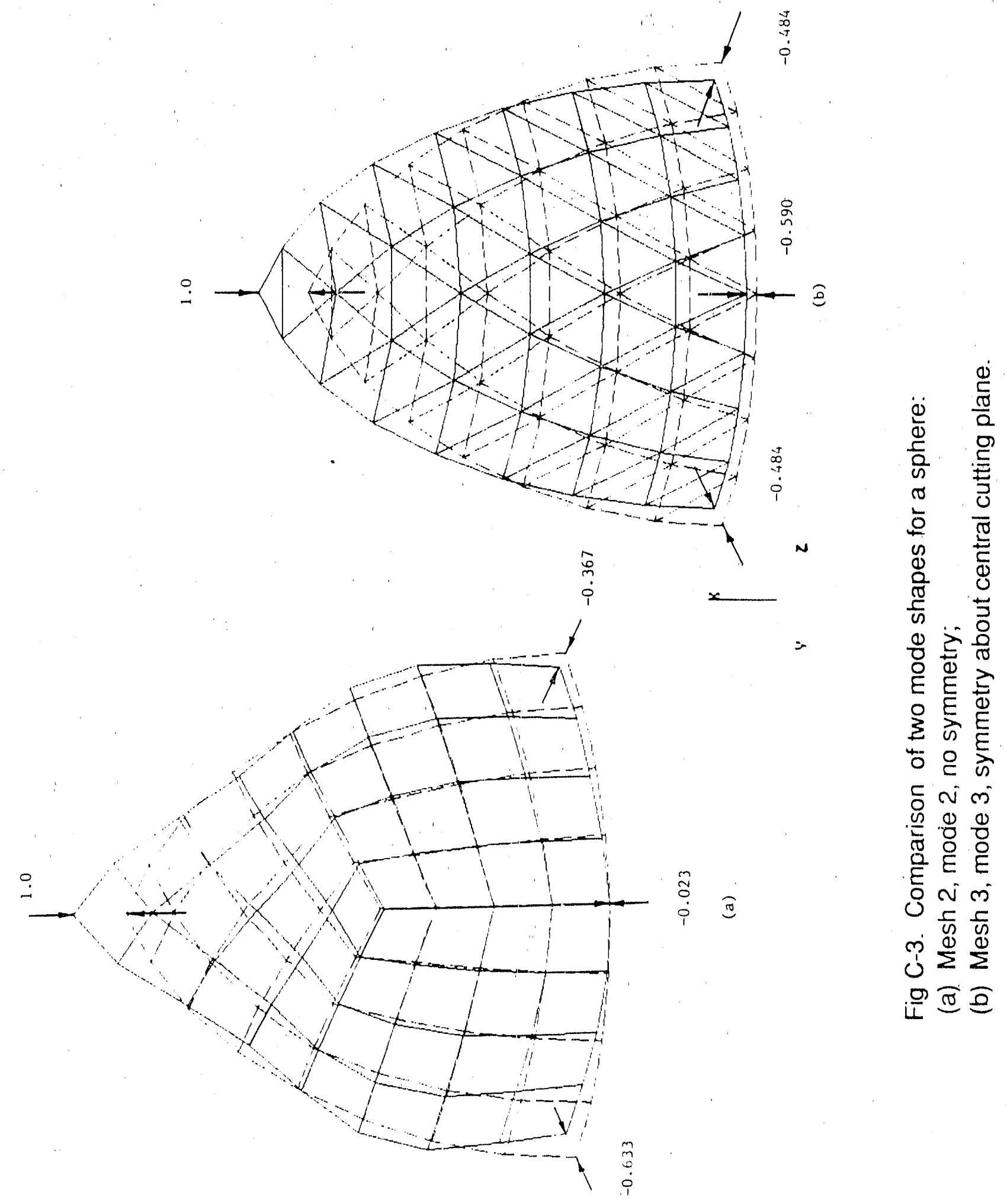


- END-

DATE FILMED

$16 / 90$ 
